\title{
On constructing RAGS via homogeneous splines
}

\author{
Carolina Vittoria Beccari ${ }^{\mathrm{a}, *}$, Marian Neamtu ${ }^{\mathrm{b}}$ \\ ${ }^{a}$ Department Mathematics, University of Bologna, P.zza di Porta San Donato 5, 40126 Bologna, Italy \\ ${ }^{b}$ Center for Constructive Approximation, Department of Mathematics, Vanderbilt University, 1326 Stevenson Center, \\ Nashville, TN 37240, U.S.A.
}

\begin{abstract}
Recently, a construction of spline spaces suitable for representing smooth parametric surfaces of arbitrary topological genus and arbitrary order of continuity has been proposed. These splines, called RAGS (rational geometric splines), are a direct generalization of bivariate polynomial splines on planar triangulations. In this paper we discuss how to construct parametric splines associated with the three homogeneous geometries (spherical, affine, and hyperbolic) and we also consider a number of related computational issues. We then show how homogeneous splines can be used to obtain RAGS. As examples of RAGS surfaces we consider direct analogs of the Powell-Sabin macro-elements and also spline surfaces of higher degrees and higher orders of continuity obtained by minimizing an energy functional.
\end{abstract}

Keywords: Splines on triangulations, Piecewise rational function, Unstructured mesh, Arbitrary topological genus, Homogeneous geometry, Homogeneous spline

2010 MSC: 65D07, 65D17

\section{Introduction}

In a recent paper [1], we have proposed a general method of defining rational splines, so-called RAGS (or rational geometric splines), capable of representing surfaces of general topological genus. The main advantage of RAGS surfaces in comparison to alternative representations, for example those based on socalled manifold splines $[2,3,4,5,6]$, subdivision methods $[7,8,9,10]$, and T-splines $[11,12,13]$, is that they mimic the standard bivariate splines on planar domains and their constructive aspects, and as such, they are closer in spirit to the traditional NURBS representation. The point of departure for constructing RAGS is a given mesh, which is a triangulation in $\mathbb{R}^{3}$ and an appropriate collection of so-called transition maps that are linear rational transformations. Such transformations allow one to "glue" together neighboring triangles of the mesh and have also been recently considered in $[14,15]$. As was pointed out in our mentioned paper, a possible way of finding such transition maps is by means of a homogeneous geometry. Our purpose in the current paper is to give more details on this approach and also to report on our initial numerical experience with RAGS.

Before we delve into details, we point out that the idea of using homogeneous geometry in connection with defining splines is not new. One attempt in this direction is reported in $[16,17,18]$, where the authors consider the so-called spherical splines, which are in essence piecewise spherical harmonic functions. In [19], such spherical splines are also used to define a class of $C^{1}$ and $C^{2}$ interpolating splines on the sphere that are piecewise rational functions and, as it turns out, they represent special instances of RAGS. Another kind of homogeneous geometry, namely the hyperbolic geometry, can be used to model surfaces of genus larger than one. This was initially noticed in [20] and later also considered in [21, 22]. It was also pointed out in

\footnotetext{
* Corresponding author

Email addresses: carolina.beccari2@unibo.it (Carolina Vittoria Beccari), mike.neamtu@gmail.com (Marian Neamtu)
} 
these last two references that such surfaces can be parameterized as piecewise rational surfaces. For a more detailed historical perspective on these topics, we refer to the discussion in [1].

The paper is organized as follows. The first part is concerned with the construction of parametric homogeneous splines and a number of algorithmic issues. Then we describe several specific interpolation and approximation methods for constructing smooth splines, including analogs of the Powell-Sabin method and a global energy-minimization method. Finally, we show how homogeneous splines can be used to obtain RAGS and present a few numerical examples.

\section{Definition of splines via homogeneous geometry}

The starting point for the construction of splines in this paper is a mesh $\Delta$. We assume that $\Delta$ is a triangulation of a finite set of points in $\mathbb{R}^{3}$. Thus, $\Delta$ will be thought of as a surface or, more precisely, a closed 2-manifold (orientable or non-orientable) embedded in $\mathbb{R}^{3}$. The assumption that $\Delta$ has no boundary is not essential, it merely serves here to simplify the exposition. Depending on its genus, this surface can be equipped with one of the standard homogeneous geometries, spherical, affine, or hyperbolic. This means that one can find a triangulation, denoted by $\Delta_{H}$, which is combinatorially equivalent to $\Delta$, corresponding to one of these homogeneous geometries (hence the subscript " $H$ " in the notation). In particular, $\Delta_{H}$ will be a collection of triangles that are either spherical (if the genus $g$ is 0 ), planar (or affine, $g=1$ ), or hyperbolic $\left(g>1\right.$.) More precisely, "triangles" in $\Delta_{H}$ will be equivalence classes of congruent triangles. To work with such triangles, we will either select a specific representative, i.e. a triple of vertices in one of the three homogeneous planes (denoted by the generic symbol $\mathcal{P}$, so that $\mathcal{P}$ will either be the sphere, the affine plane, or the hyperbolic plane), or we will represent such a triangle as a triple of its interior angles.

In order for $\Delta_{H}$ to be a well-defined triangulation, triangles in $\Delta_{H}$ must be compatible in the following sense. Let $v$ be a vertex of $\Delta$ and let $C(v)$ be the set of all triangles $T_{1}, \ldots, T_{\nu} \in \Delta$ containing $v$. We call $C(v)$ the cell corresponding to vertex $v$. Here $\nu$ denotes the valence of $v$, i.e., the cardinality of $C(v)$. Without loss of generality, we will assume that the triangles of $C(v)$ are ordered such that $T_{1}=\left\langle v, v_{1}, v_{2}\right\rangle, \ldots, T_{\nu-1}=$ $\left\langle v, v_{\nu-1}, v_{\nu}\right\rangle, T_{\nu}=\left\langle v, v_{\nu}, v_{1}\right\rangle$, where $v_{1}, \ldots, v_{\nu}$ are the vertices of $\Delta$ that are connected to $v$ by an edge of $\Delta$. Next, let $\mathcal{C}(v)=\left\{\mathcal{T}_{1}, \ldots, \mathcal{T}_{\nu}\right\}$ be the set of triangles in $\Delta_{H}$ corresponding to $C(v)$. The mentioned compatibility condition on $\Delta_{H}$ is that there must exist a set of points $u, u_{1}, \ldots, u_{\nu} \in \mathcal{P}$, such that triangles $\left\langle u, u_{1}, u_{2}\right\rangle, \ldots,\left\langle u, u_{\nu}, u_{1}\right\rangle$ have disjoint interiors (i.e. are non-overlapping) and are congruent to $\mathcal{T}_{1}, \ldots, \mathcal{T}_{\nu}$, respectively. Thus, we can think of $\mathcal{C}(v)$ as a set of physical triangles in $\mathcal{P}$, sharing a common vertex $u$.

The above compatibility condition implies the following fact, to be used later. Let $T, T^{\prime}$ be adjacent triangles of $\Delta$, i.e. such that they share a common edge, and let $\mathcal{T}, \mathcal{T}^{\prime}$ be their corresponding counterparts in $\Delta_{H}$ (we also call such $\mathcal{T}, \mathcal{T}^{\prime}$ adjacent.) Then one can find four points $u_{1}, u_{2}, u_{3}, u_{3}^{\prime}$ such that the triangles $\left\langle u_{1}, u_{2}, u_{3}\right\rangle,\left\langle u_{1}, u_{2}, u_{3}^{\prime}\right\rangle$ do not overlap and that they are congruent with $\mathcal{T}, \mathcal{T}^{\prime}$, respectively.

To explain the construction of a spline space corresponding to $\Delta$ (or, equivalently, to $\Delta_{H}$ ), we first consider a single triangle $\mathcal{T}$ in $\Delta_{H}$ and define appropriate spaces of "polynomials" that will be used as building blocks for the desired splines.

\subsection{Homogeneous barycentric coordinates}

Given three linearly independent vectors $u_{1}, u_{2}, u_{3} \in \mathbb{R}^{3}$, any vector $u \in \mathbb{R}^{3}$ can be expressed in a unique way as a linear combination $u=b_{1}(u) u_{1}+b_{2}(u) u_{2}+b_{3}(u) u_{3}$. The numbers $b_{1}, b_{2}, b_{3}$ are linear homogeneous functions of $u$ and are called the trihedral or homogeneous barycentric coordinates of $u$ with respect to $u_{1}, u_{2}, u_{3}$ [17]. Recall that a function $f$ defined on $\mathbb{R}^{3}$ is said to be homogeneous of degree $n$ provided $f(\alpha v)=\alpha^{n} f(v), \alpha \in \mathbb{R}, v \in \mathbb{R}^{3}$. Thus, the coordinates $b_{1}, b_{2}, b_{3}$ are trivariate homogeneous polynomials of degree one.

In this paper we will be interested in the cases where the points $u_{1}, u_{2}, u_{3}$ are vertices of a triangle in one of the three homogeneous planes $\mathcal{P}$. In particular, we assume that $\mathcal{P}$ is one of the standard surfaces, i.e. either the sphere, $\mathcal{S}=\left\{(x, y, z) \in \mathbb{R}^{3} \mid x^{2}+y^{2}+z^{2}=1\right\}$, the affine plane, $\mathcal{A}=\left\{(x, y, z) \in \mathbb{R}^{3} \mid z=1\right\}$, or the hyperbolic plane, $\mathcal{H}=\left\{(x, y, z) \in \mathbb{R}^{3} \mid-x^{2}-y^{2}+z^{2}=1, z>0\right\}$, and we will consider the restrictions of $b_{1}, b_{2}, b_{3}$ to $\mathcal{P}$. In the first case, these restrictions have been termed spherical barycentric coordinates [16], in 
the second case, one obtains the standard planar or affine coordinates (since $\left.\left.\left(b_{1}+b_{2}+b_{3}\right)\right|_{\mathcal{A}}=1\right)$, and, lastly, if $\mathcal{P}=\mathcal{H}$, the restrictions could be dubbed hyperbolic barycentric coordinates. However, for simplicity, we will use the generic term barycentric coordinates, or b.c.s, for short.

Barycentric coordinates share many properties with their planar counterparts. Let $u_{1}, u_{2}, u_{3} \in \mathcal{P}$ be linearly independent and let $V\left(u_{1}, u_{2}, u_{3}\right):=\left\{u \in \mathbb{R}^{3}: b_{1}(u), b_{2}(u), b_{3}(u) \geqslant 0\right\}$ be the corresponding trihedron. By $\mathcal{T}=\left\langle u_{1}, u_{2}, u_{3}\right\rangle \subset \mathcal{P}$, we denote the triangle obtained as the intersection of $V\left(u_{1}, u_{2}, u_{3}\right)$ with $\mathcal{P}$. We list the following facts without proof. For $i=1,2,3$, one has

(P1) $b_{i}\left(u_{j}\right)=\delta_{i j}, j=1,2,3$, where $\delta_{i j}$ is the Kronecker delta;

(P2) $b_{i}(u) \geqslant 0, u \in \mathcal{T}$;

(P3) $b_{i}(u)=0$, for $u \in \mathcal{P}$, if and only if $u$ is on the geodesic containing edge $\varepsilon_{i}$ of $\mathcal{T}$ opposite to $u_{i}$. In the canonical setting, where $\mathcal{P}$ is one of the three standard surfaces considered above, a geodesic is the intersection of $\mathcal{P}$ with a plane in $\mathbb{R}^{3}$ passing through the origin;

(P4) for $u \in \mathcal{T} \backslash\left\{u_{1}, u_{2}, u_{3}\right\}$, one has $b_{1}(u)+b_{2}(u)+b_{3}(u) \begin{cases}>1, & \mathcal{P}=\mathcal{S}, \\ =1, & \mathcal{P}=\mathcal{A}, \\ <1, & \mathcal{P}=\mathcal{H}\end{cases}$

Property (P3) implies that a point $u$ on an edge of $\mathcal{T}$ can be expressed by means of its b.c.s with respect to the endpoints of that edge, or $u=b_{1} u_{1}+b_{2} u_{2}, u \in \widehat{u_{1} u_{2}}$, where $\widehat{u_{1} u_{2}}$ denotes the edge (or geodesic arc) of $\mathcal{T}$ with endpoints $u_{1}, u_{2}$. The next three propositions provide means for the evaluation of b.c.s. The following result is stated without proof (but see [16] for the spherical case).
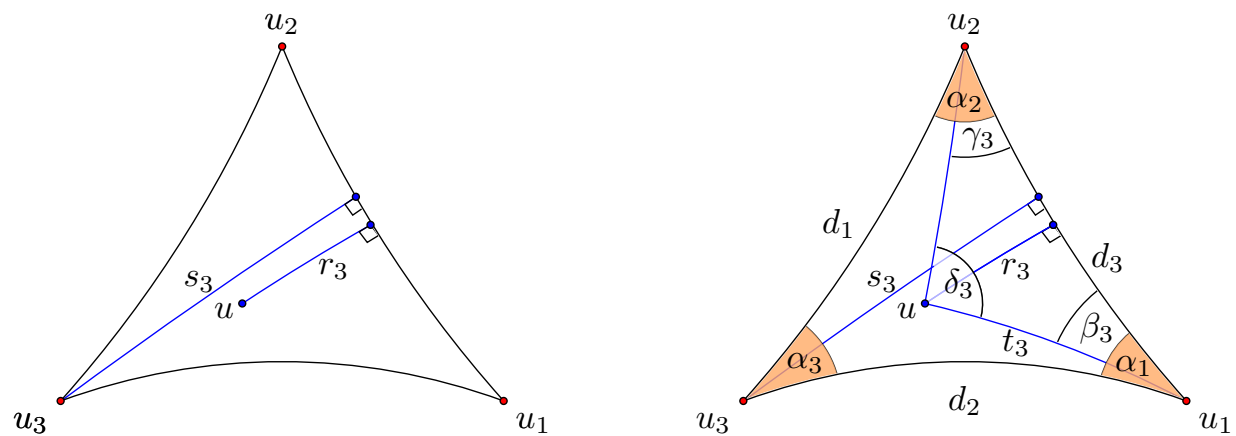

Figure 1: Notation used in Propositions 1 and 2, depicting the case of hyperbolic triangles.

Proposition 1. Let $\mathcal{T}=\left\langle u_{1}, u_{2}, u_{3}\right\rangle \subset \mathcal{P}$ be a non-degenerate triangle, i.e. such that $u_{1}, u_{2}, u_{3}$ are independent, and let $u \in \mathcal{P}$. Then

$$
b_{i}(u)=\frac{\rho\left(r_{i}\right)}{\rho\left(s_{i}\right)}, \quad i=1,2,3,
$$

where $r_{i}, s_{i}$ are the respective signed distances of $u, u_{i}$ from the geodesic containing edge $\varepsilon_{i}$ (Fig. 1(left)) and

$$
\rho(t):= \begin{cases}\sin (t), & \mathcal{P}=\mathcal{S} \\ t, & \mathcal{P}=\mathcal{A} \\ \sinh (t), & \mathcal{P}=\mathcal{H} .\end{cases}
$$

We use the convention that $s_{i}>0$ and that $r_{i}<0$, if $u, u_{i}$ are on the opposite sides of the plane passing through $\varepsilon_{i}$ and the origin, and $r_{i} \geqslant 0$ otherwise.

Another useful property of b.c.s is that they are invariant under congruence transformations. This can be seen as a direct consequence of the following formulas for b.c.s., showing that the b.c.s depend only on internal angles of associated triangles. 
Proposition 2. Let $\mathcal{T}=\left\langle u_{1}, u_{2}, u_{3}\right\rangle \subset \mathcal{P}$ be non-degenerate and $u \in \mathcal{P}$. Then

$$
b_{i}(u)=\frac{\sin \alpha_{i} \sin \beta_{i} \sin \gamma_{i}}{\sin \delta_{i} \sin \alpha_{i-1} \sin \alpha_{i+1}}, \quad i=1,2,3,
$$

where the expression on the right is defined as 0 if $\delta_{i}=\pi$, and where we assume that the indices $i-1, i, i+1$ are a cyclic permutation of $1,2,3$. Here we denoted by $\beta_{i}, \gamma_{i}, \delta_{i}$ the interior angles of the triangle $\left\langle u_{i+1}, u_{i-1}, u\right\rangle$, see Fig. 1(right).

Proof. We adopt the convention that $\beta_{i}, \gamma_{i} \in[0, \pi], \delta_{i} \in(0, \pi]$ if $u$ and $u_{i}$ are on the same side of edge $\widehat{u_{i-1} u_{i+1}}$, whereas $\beta_{i}, \gamma_{i} \in[\pi, 2 \pi], \delta_{i} \in[\pi, 2 \pi)$ otherwise. We will prove the statement for the case $i=3$, the other cases being similar. If $u$ is on the geodesic containing edge $\varepsilon_{3}=\widehat{u_{1} u_{2}}$, or $\delta_{3}=\pi$, then by property (P3), $b_{3}(u)=0$. Otherwise, identity (2) can be proved by a repeated application of the law of sines. In particular, for triangle $\left\langle u_{1}, u_{2}, u\right\rangle$ and one of its subtriangles, we get

$$
\frac{\rho\left(t_{3}\right)}{\rho\left(d_{3}\right)}=\frac{\sin \gamma_{3}}{\sin \delta_{3}}, \quad \frac{\rho\left(r_{3}\right)}{\rho\left(t_{3}\right)}=\frac{\sin \beta_{3}}{\sin (\pi / 2)},
$$

whereas for triangle $\left\langle u_{1}, u_{2}, u_{3}\right\rangle$ and one of its subtriangles, one has

$$
\frac{\rho\left(d_{2}\right)}{\rho\left(d_{3}\right)}=\frac{\sin \alpha_{2}}{\sin \alpha_{3}}, \quad \frac{\rho\left(s_{3}\right)}{\rho\left(d_{2}\right)}=\frac{\sin \alpha_{1}}{\sin (\pi / 2)}
$$

Formula (1), together with the above identities, yields the desired equality

$$
b_{3}(u)=\frac{\rho\left(r_{3}\right)}{\rho\left(s_{3}\right)}=\frac{\sin \alpha_{3} \sin \beta_{3} \sin \gamma_{3}}{\sin \delta_{3} \sin \alpha_{1} \sin \alpha_{2}}
$$

which completes the proof.

The result above allows us to prove the following important formulas for b.c.s. associated with adjacent triangles.

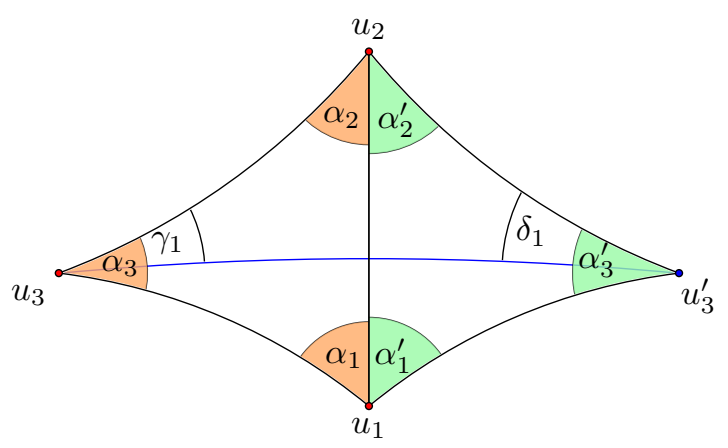

Figure 2: Notation used in Proposition 3.

Proposition 3. Consider two adjacent triangles $\mathcal{T}=\left\langle u_{1}, u_{2}, u_{3}\right\rangle$ and $\mathcal{T}^{\prime}=\left\langle u_{1}, u_{2}, u_{3}^{\prime}\right\rangle$ in $\mathcal{P}$, with angles $\left\langle\alpha_{1}, \alpha_{2}, \alpha_{3}\right\rangle$ and $\left\langle\alpha_{1}^{\prime}, \alpha_{2}^{\prime}, \alpha_{3}^{\prime}\right\rangle$. Let $\lambda_{1}, \lambda_{2}, \lambda_{3}$ be the b.c.s of $u_{3}^{\prime}$ w.r.t. $\mathcal{T}$. Then

$$
\lambda_{1}=\frac{\sin \left(\alpha_{2}+\alpha_{2}^{\prime}\right) \sin \alpha_{1} \sin \alpha_{1}^{\prime}}{\sin \alpha_{3}^{\prime} \sin \alpha_{1} \sin \alpha_{2}}, \quad \lambda_{2}=\frac{\sin \left(\alpha_{1}+\alpha_{1}^{\prime}\right) \sin \alpha_{2} \sin \alpha_{2}^{\prime}}{\sin \alpha_{3}^{\prime} \sin \alpha_{1} \sin \alpha_{2}}, \quad \lambda_{3}=-\frac{\sin \alpha_{3} \sin \alpha_{1}^{\prime} \sin \alpha_{2}^{\prime}}{\sin \alpha_{3}^{\prime} \sin \alpha_{1} \sin \alpha_{2}}
$$

Proof. We follow the notation in Fig. 2, where angles $\gamma_{1}, \delta_{1}$ are the interior angles of triangle $\left\langle u_{2}, u_{3}, u_{3}^{\prime}\right\rangle$ corresponding to vertices $u_{3}, u_{3}^{\prime}$, respectively. The expression for $\lambda_{3}$ is an immediate consequence of Proposition 2 , with $i=3, u=u_{3}^{\prime}, \delta_{3}=2 \pi-\alpha_{3}^{\prime}, \beta_{3}=2 \pi-\alpha_{1}^{\prime}, \gamma_{3}=2 \pi-\alpha_{2}^{\prime}$. As for $\lambda_{1}$, suppose first that $\delta_{1}=0$. 
Then the three points $u_{2}, u_{3}, u_{3}^{\prime}$ lie on a single geodesic and hence, by property (P3), $\lambda_{1}=0$. On account of $\alpha_{2}+\alpha_{2}^{\prime}=\pi$, this is consistent with the first identity in (3). Next, we proceed under the assumption that $\delta_{1} \neq 0$. Then formula (2), with $i=1, \beta_{1}=\alpha_{2}+\alpha_{2}^{\prime}$, gives

$$
\lambda_{1}=\frac{\sin \alpha_{1} \sin \left(\alpha_{2}+\alpha_{2}^{\prime}\right) \sin \gamma_{1}}{\sin \delta_{1} \sin \alpha_{2} \sin \alpha_{3}} .
$$

Therefore, to prove the assertion of the proposition, it remains to show that

$$
\frac{\sin \alpha_{1} \sin \gamma_{1} \sin \alpha_{3}^{\prime}}{\sin \delta_{1} \sin \alpha_{3} \sin \alpha_{1}^{\prime}}=1
$$

However, this can be easily verified by applying the law of sines to triangles $\left\langle u_{3}, u_{3}^{\prime}, u_{2}\right\rangle,\left\langle u_{1}, u_{2}, u_{3}\right\rangle$, and $\left\langle u_{1}, u_{2}, u_{3}^{\prime}\right\rangle$. The proof for $\lambda_{2}$ is analogous.

The above proposition states that the interior angles associated with triangles $\mathcal{T}, \mathcal{T}^{\prime}$ readily determine the b.c.s $\lambda_{1}, \lambda_{2}, \lambda_{3}$. This is not surprising, given the mentioned fact that the b.c.s are invariant under congruence transformations. Curiously, the formula is formally the same in all three cases (spherical, affine, hyperbolic.) Moreover, the expressions for $\lambda_{1}, \lambda_{2}, \lambda_{3}$ are always well defined as long as the two triangles are non-degenerate, i.e., all associated angles are in $(0, \pi)$. Lastly, in the affine case one has $\alpha_{1}+\alpha_{2}+\alpha_{3}=$ $\alpha_{1}^{\prime}+\alpha_{2}^{\prime}+\alpha_{3}^{\prime}=\pi$ and it is easy to see that then $\lambda_{1}+\lambda_{2}+\lambda_{3}=1$, as expected.

\subsection{Homogeneous Bernstein-Bézier polynomials}

We recall some elementary facts concerning homogeneous polynomials [16]. Let $\mathcal{T}=\left\langle u_{1}, u_{2}, u_{3}\right\rangle$ be a nondegenerate triangle in $\mathcal{P}$ and let $b_{1}, b_{2}, b_{3}$ denote the associated b.c.s. Homogeneous Bernstein basis polynomials of degree $n$ are defined as

$$
B_{i j k}^{n}(u):=\frac{n !}{i ! j ! k !} b_{1}^{i}(u) b_{2}^{j}(u) b_{3}^{k}(u), \quad i+j+k=n, \quad u \in \mathcal{P} .
$$

It can be easily proved that such functions are linearly independent and hence every $p$ in $\pi_{n}$, the space of homogeneous parametric polynomials of degree $n$, has a unique expansion of the form

$$
p(u)=\sum_{i+j+k=n} c_{i j k} B_{i j k}^{n}(u), \quad u \in \mathcal{P},
$$

where the $c_{i j k} \in \mathbb{R}^{3}$ are the so-called Bézier- or control points of $p$. This equation is customarily referred to as the Bernstein-Bézier representation, or simply the $B$-form, of $p$.

Due to their formal analogy with ordinary bivariate polynomials, homogeneous polynomials in the Bform inherit many of the properties of their planar counterparts. In particular, they can be evaluated by means of the well-known de Casteljau algorithm and conditions for two polynomials on adjacent triangles joining smoothly across their common edge are formally the same as those for ordinary polynomials, as can be seen below. We will omit the proof of the next result since it follows along standard lines (see e.g. [16]).

Proposition 4. Let $\mathcal{T}=\left\langle u_{1}, u_{2}, u_{3}\right\rangle$ and $\mathcal{T}^{\prime}=\left\langle u_{1}, u_{2}, u_{3}^{\prime}\right\rangle$ be two adjacent triangles in $\mathcal{P}$ and let

$$
p=\sum_{i+j+k=n} c_{i j k} B_{i j k}^{n} \quad \text { and } \quad p^{\prime}=\sum_{i+j+k=n} c_{i j k}^{\prime} B_{i j k}^{\prime n}
$$

be polynomials defined on $\mathcal{T}$ and $\mathcal{T}^{\prime}$, respectively. Then $p$ and $p^{\prime}$ join with $C^{m}$ continuity, $m=0, \ldots, n$, along the common edge $\widehat{u_{1} u_{2}}$ if and only if

$$
c_{i j k}^{\prime}=\sum_{\nu+\mu+\kappa=k} c_{i+\nu, j+\mu, k} B_{\nu \mu \kappa}^{k}\left(u_{3}^{\prime}\right), \quad k=0, \ldots, m, \quad i+j+k=n .
$$


Thus, in particular, $C^{0}$-continuity conditions for $p, p^{\prime}$ read:

$$
c_{i j 0}^{\prime}=c_{i j 0}, \quad i+j=n,
$$

whereas $C^{1}$-continuity conditions take the familiar form:

$$
c_{i j 1}^{\prime}=\lambda_{1} c_{i+1, j, 0}+\lambda_{2} c_{i, j+1,0}+\lambda_{3} c_{i j 1}, \quad i+j=n-1,
$$

where $\lambda_{1}, \lambda_{2}, \lambda_{3}$ are the b.c.s of $u_{3}^{\prime}$ w.r.t. $u_{1}, u_{2}, u_{3}$, which can be obtained by formulas in Proposition 3 .

\subsection{Homogeneous splines}

Homogeneous polynomials defined in the previous section can be used to construct spline spaces corresponding to a given mesh $\Delta$ by means of the combinatorially equivalent triangulation $\Delta_{H}$. A spline $s$ in such a space will simply be a piecewise-defined function composed of individual polynomial pieces $s^{\mathcal{T}}:=\left.\left.s\right|_{\mathcal{T}} \in \pi_{n}\right|_{\mathcal{T}}$, where $\mathcal{T}$ will vary over the triangles of $\Delta_{H}$. Note that by definition (4), for given fixed Bézier coefficients $c_{i j k}$ of $s^{\mathcal{T}}$, this function will not depend on which particular representative of the equivalence class of congruent triangles with $\mathcal{T}$ is considered, given that b.c.s are invariant under congruence transformations. We will use the notation $s: \Delta_{H} \rightarrow \mathbb{R}^{3}$ for composite parametric functions $s$ such that, for any $\mathcal{T} \in \Delta_{H}$, the restriction $s^{\mathcal{T}}$ satisfies $s^{\mathcal{T}}(u)=\hat{s}(b(u)), u \in \mathcal{T}$, for some function $\hat{s}$ independent of the particular representative chosen for $\mathcal{T}$, where $b(u)$ is the vector of b.c.s of $u$ w.r.t. $\mathcal{T}$.

In addition to being piecewise (homogeneous) polynomials, our splines will be required to be globally $C^{m}$ continuous, for some $m \geq 0$, i.e. they will belong to the class $C^{m}\left(\Delta_{H}\right)$. This will be the space of all functions $f: \Delta_{H} \rightarrow \mathbb{R}^{3}$ that are $C^{m}$ continuous in the following sense. Let $v$ be a vertex of $\Delta$ and let $\mathcal{C}(v)$ be the cell of $\Delta_{H}$ corresponding to cell $C(v)$ of $\Delta$, whose existence was postulated at the beginning of Section 2. Also, let $\mathcal{U}(v) \subset \mathcal{P}$ be the union of all triangles of $\mathcal{C}(v)$. We say that a function $f: \Delta_{H} \rightarrow \mathbb{R}^{3}$ is $C^{m}$ continuous if $f$ is $C^{m}$ continuous in the interior of $\mathcal{U}(v)$, for all vertices $v$ of $\Delta$. We note that $f$ is well defined in all of $\mathcal{U}(v)$ since it is defined for all $\mathcal{T} \in \mathcal{C}(v)$ and values of $\left.f\right|_{\mathcal{T}}$ do not depend on the particular representative for $\mathcal{T}$ that we choose (since $\left.f\right|_{\mathcal{T}}$ is a function of b.c.s).

We are now ready to define a spline space of piecewise homogeneous polynomial functions associated with $\Delta$. Let $m, n$ be nonnegative integers. We call

$$
S_{n}^{m}(\Delta):=\left\{s \in C^{m}\left(\Delta_{H}\right):\left.\left.s\right|_{\mathcal{T}} \in \pi_{n}\right|_{\mathcal{T}}, \mathcal{T} \in \Delta_{H}\right\}
$$

the space of homogeneous splines of degree $n$ and smoothness $m$. We will refer to these spaces as spherical, affine, and hyperbolic splines in the cases $\mathcal{P}=\mathcal{S}, \mathcal{A}, \mathcal{H}$, respectively.

To construct a spline for a given triangulation $\Delta$, we will first need to find an appropriate triangulation $\Delta_{H}$. This will be discussed in the next section. After that continuity conditions will be imposed between neighboring Bézier patches (i.e. polynomials associated with adjacent triangles $\mathcal{T}, \mathcal{T}^{\prime}$ ), specified in Proposition 4, which will give rise to a linear system of equations for the Bézier points of the individual patches.

Of immediate interest is the problem of the dimension of the space $S_{n}^{m}(\Delta)$. This is known to be a difficult question even in the affine case and, in fact, the exact dimension is known only when $n$ is sufficiently large relative to $m$, see the extensive discussion in [23]. In the non-affine case, the situation is similar. For example, the spherical case has been dealt with in detail in [17] and we expect that in the hyperbolic case the results will be similar, especially in the tractable case $n \geq 3 m+2$, since, as was pointed out earlier, smoothness conditions between neighboring polynomial pieces are formally the same for all three types of geometries. However, the dimension question and the construction of bases for $S_{n}^{m}(\Delta)$ will not be dealt with in this paper.

Here we will content ourselves with stating the dimension in the special case of $C^{0}$ splines. To this end we let $n_{V}, n_{E}, n_{T}$ denote the respective numbers of vertices, edges, and triangles of $\Delta$. Then

$$
\operatorname{dim} S_{n}^{0}(\Delta)=n_{V}+(n-1) n_{E}+\frac{1}{2}(n-1)(n-2) n_{T},
$$


which is a direct consequence of the $C^{0}$-continuity conditions (5) and the fact that the Bernstein basis polynomials in (4) are independent and in one-to-one correspondence with control points $c_{i j k}$. We also note in passing the following well-known formulas for the numbers $n_{V}, n_{E}, n_{T}$, and the genus $g$ of $\Delta$ :

$$
n_{T}=2 n_{V}+4(g-1), \quad n_{E}=3 n_{V}+6(g-1) .
$$

\section{Computing triangulation $\Delta_{H}$}

Given the initial triangulation $\Delta$, the definition of the spline space in previous section requires the knowledge of a triangulation $\Delta_{H}$. In particular, there is a one-to-one correspondence between triangles in $\Delta$ and $\Delta_{H}$. We will use the notation $\Delta=\left\{T_{i}\right\}_{i=1}^{n_{T}}$ and $\Delta_{H}=\left\{\mathcal{T}_{i}\right\}_{i=1}^{n_{T}}$.

In this section we will introduce a possible way to obtain $\Delta_{H}$. Since elements of $\Delta_{H}$ are equivalence classes of congruent triangles in $\mathcal{P}$, we will assume that triangles in $\Delta_{H}$ are represented as triples of angles rather than triples of points in $\mathcal{P}$. We will henceforth use the notation $\mathcal{T}_{i}=\left\langle\alpha_{1}^{i}, \alpha_{2}^{i}, \alpha_{3}^{i}\right\rangle$, where $\alpha_{1}^{i}, \alpha_{3}^{i}, \alpha_{3}^{i} \in(0, \pi)$. The lengths of the edges opposite to these angles will be denoted by $d_{1}^{i}, d_{2}^{i}, d_{3}^{i}$. It is well known that in the spherical case one has

$$
\cos d_{3}^{i}=\frac{\cos \alpha_{1}^{i} \cos \alpha_{2}^{i}+\cos \alpha_{3}^{i}}{\sin \alpha_{1}^{i} \sin \alpha_{2}^{i}}
$$

whereas in the hyperbolic case the same formula holds, with $\cos d_{3}^{i}$ replaced with $\cosh d_{3}^{i}$ [24]. Formulas for $d_{1}^{i}, d_{2}^{i}$ are analogous.

We also recall that any triple $\alpha_{1}^{i}, \alpha_{2}^{i}, \alpha_{3}^{i} \in(0, \pi)$ gives rise to a unique triangle (up to congruence) in one of the three homogeneous geometries, namely to a hyperbolic triangle, if $\alpha_{1}^{i}+\alpha_{2}^{i}+\alpha_{3}^{i}<\pi$, or affine, if $\alpha_{1}^{i}+\alpha_{2}^{i}+\alpha_{3}^{i}=\pi$, or spherical, if $\alpha_{1}^{i}+\alpha_{2}^{i}+\alpha_{3}^{i}>\pi$.

The following are necessary and sufficient conditions on angles $\alpha_{1}^{i}, \alpha_{2}^{i}, \alpha_{3}^{i}$, for the existence of a consistent triangulation $\Delta_{H}$ :

(T1) the sum of angles around each vertex is $2 \pi$;

(T2) common sides of adjacent triangles have equal lengths.

These conditions guarantee that all triangles are properly "glued together" and with no "holes". It is well known that such a triangulation $\Delta_{H}$ always exists [24]. Moreover, a consequence of the Gauss-Bonnet Theorem is the following identity involving the genus $g$ of $\Delta$ :

$$
\sum_{i=1}^{n_{T}}\left(\pi-\left(\alpha_{1}^{i}+\alpha_{2}^{i}+\alpha_{3}^{i}\right)\right)=4 \pi(g-1) .
$$

The problem of finding a suitable triangulation $\Delta_{H}$ can be thought of as a parametrization problem and it has received considerable attention in the planar case, see for example [25]. In the following we describe the algorithm we have used in our examples, although alternative approaches are possible.

We use the convention that all angles surrounding vertex $j$ of valence $\nu_{j}$ are labeled as $\beta_{j}^{i_{k}}$, where $i_{k}$ is the index of the $k$-th triangle containing vertex $j$, and where $k=1, \ldots, \nu_{j}$ (Fig. 3(left)). We will seek a set of appropriate angles defining $\Delta_{H}$ as a solution of a constrained minimization problem with a suitable objective function and with nonlinear constraints represented by conditions (T1) and (T2). A possibility is to minimize the quadratic function

$$
\sum_{i=1}^{n_{T}}\left(\alpha_{1}^{i}-\alpha_{2}^{i}\right)^{2}+\left(\alpha_{2}^{i}-\alpha_{3}^{i}\right)^{2}+\left(\alpha_{3}^{i}-\alpha_{1}^{i}\right)^{2},
$$

so as to obtain a triangulation which is as close as possible to being "uniform". Moreover, by (7), condition (T2) gives rise to a set of distance constraints:

$$
d_{i j}:=\frac{\cos \alpha_{1}^{i} \cos \alpha_{2}^{i}+\cos \alpha_{3}^{i}}{\sin \alpha_{1}^{i} \sin \alpha_{2}^{i}}-\frac{\cos \alpha_{1}^{j} \cos \alpha_{2}^{j}+\cos \alpha_{3}^{j}}{\sin \alpha_{1}^{j} \sin \alpha_{2}^{j}}=0,
$$


for all pairs of adjacent triangles $\mathcal{T}_{i}=\left\langle\alpha_{1}^{i}, \alpha_{2}^{i}, \alpha_{3}^{i}\right\rangle, \mathcal{T}_{j}=\left\langle\alpha_{1}^{j}, \alpha_{2}^{j}, \alpha_{3}^{j}\right\rangle$, where angles $\alpha_{3}^{i}$ and $\alpha_{3}^{j}$ are assumed to be opposite the common edge (cf. Fig. 3(right)), whereas condition (T1) can be expressed as

$$
\sum_{k=1}^{\nu_{j}} \beta_{j}^{i_{k}}=2 \pi, \quad j=1, \ldots, n_{V}
$$
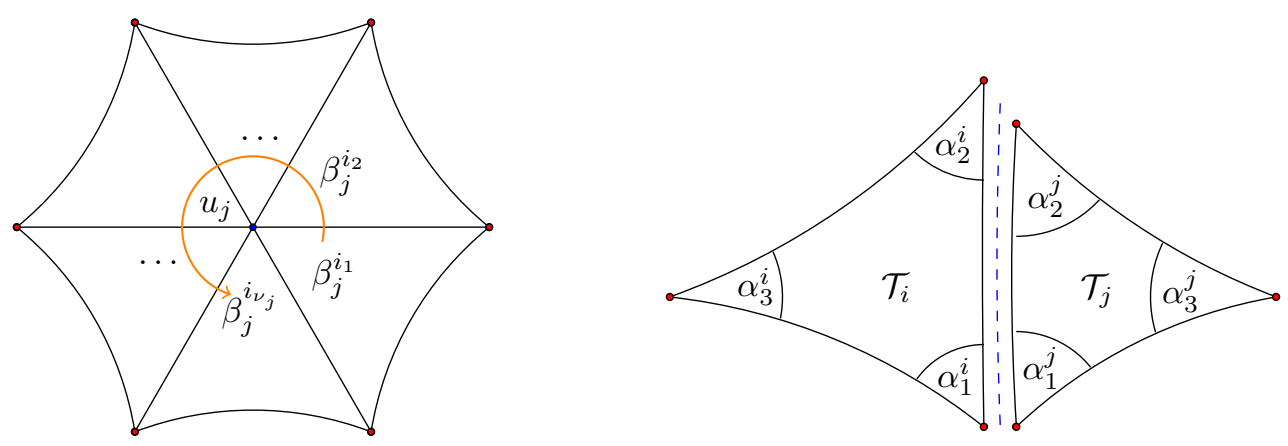

Figure 3: Configuration of angles around a vertex (left); Discrepancy in edge lengths along the common edge of two adjacent triangles $\mathcal{T}_{i}$ and $\mathcal{T}_{j}$ (right).

Equations (9) and (10) are constraints associated with vertices and edges of $\Delta_{H}$, and hence their total number is $n_{V}+n_{E}$. For meshes of moderate size, such as the one depicted later in Fig. 6 , we have successfully solved the above optimization problem by the MATLAB routine FSOLVE, using the Levenberg-Marquard algorithm, with the tolerance set to the machine precision, and with initial values

$$
\beta_{j}^{i_{k}}=\frac{2 \pi}{\nu_{j}}, \quad k=1, \ldots, \nu_{j}, \quad j=1, \ldots, n_{V} .
$$

It should be pointed out that the expression on the right-hand side of (7) is not related to the length of an edge in the affine case, i.e. when $\alpha_{1}^{i}+\alpha_{2}^{i}+\alpha_{3}^{i}=\pi$. In that case the expression equals 1 and hence condition (9) is automatically satisfied. We further note that the formulation of the optimization problem does not require the genus $g$ as input and nor is it necessary to specify which of the three homogeneous geometries is to be used. Lastly, instead of (8), it would make sense to devise more sophisticated objective functions, for example ones that take into account the geometry of the mesh $\Delta$, so as to minimize distortions in the parametrization. We view this as a separate problem of finding an optimal parametrization. Although not studied in the current paper, we think this problem deserves a thorough investigation.

\section{Surface fitting using homogeneous splines}

A distinct advantage of homogeneous splines is that standard bivariate spline methods - which are well suited for modeling (scalar and vector) functions over planar triangulations - can be adapted to surfaces of general topology without the need to invent entirely new approaches. To illustrate this point, we present in this section a few examples of local and global surface fitting methods.

Local techniques are traditionally based on the use of so-called macro-elements. As a prototype of such splines, we have chosen the classical Powell-Sabin method, although other well-known types of elements can be obtained as a direct generalization of the affine case, such as the Clough-Tocher cubic element and many others (described for example in the recent monograph [26].)

As for global methods, we describe here how to construct interpolating spline surfaces of arbitrary degrees and orders of continuity by minimizing a suitable energy functional. 


\subsection{Interpolation by Powell-Sabin macro-elements}

The Powell-Sabin scheme consists in subdividing each triangle $\mathcal{T} \in \Delta_{H}$, called in this context a macrotriangle, into six subtriangles. A quadratic polynomial is then defined on each subtriangle in such a way that the resulting composite surface is globally $C^{1}$ continuous (for more details on this construction in the planar case, see [23, 26].) To explain this further, let $\mathcal{T}=\left\langle u_{1}, u_{2}, u_{3}\right\rangle$, where $u_{1}, u_{2}, u_{3} \in \mathcal{P}$. The splitting of the triangle can be done by considering the incenter $\xi$ of $\mathcal{T}$, i.e., the intersection of the angle bisectors of $\mathcal{T}$. One way to compute the incenter is to radially project the incenter of the flat triangle with vertices $u_{1}, u_{2}, u_{3}$ onto $\mathcal{P}$. The next step in the subdivision is to split each of the three edges of $\mathcal{T}$. Let $\mathcal{T}^{\prime}$ be an adjacent triangle to $\mathcal{T}$, with common edge $\widehat{u_{1} u_{2}}$, say, and let $\xi^{\prime}$ be the incenter of $\mathcal{T}^{\prime}$. We define $\xi_{3}$ to be the intersection of $\widehat{u_{1} u_{2}}$ with the geodesic line passing through the two incenters $\xi$ and $\xi^{\prime}$. The reason for choosing incenters of $\mathcal{T}$ and $\mathcal{T}^{\prime}$ is that this guarantees that $\xi_{3}$ is strictly inside the edge $\widehat{u_{1} u_{2}}$. This follows by a similar argument as in the plane (see Lemma 4.19 in [23]). Points $\xi_{1}, \xi_{2}$ are defined analogously.

The four points $\xi, \xi_{1}, \xi_{2}, \xi_{3}$ give rise to a subdivision of $\mathcal{T}$ into six subtriangles, as shown in Fig. 4(left). To associate a quadratic Bézier polynomial with each subtriangle, it will be necessary to determine appropriate Bézier points, which will be labeled as in Fig. 4(right). These will be defined such that the resulting spline surface interpolates the vertices of $\Delta$ and is globally $C^{1}$ smooth. The standard way to achieve the latter is to also interpolate directional derivatives at the vertices of each of the macro-elements. If these derivatives are not given, which is often the case, they can be estimated.
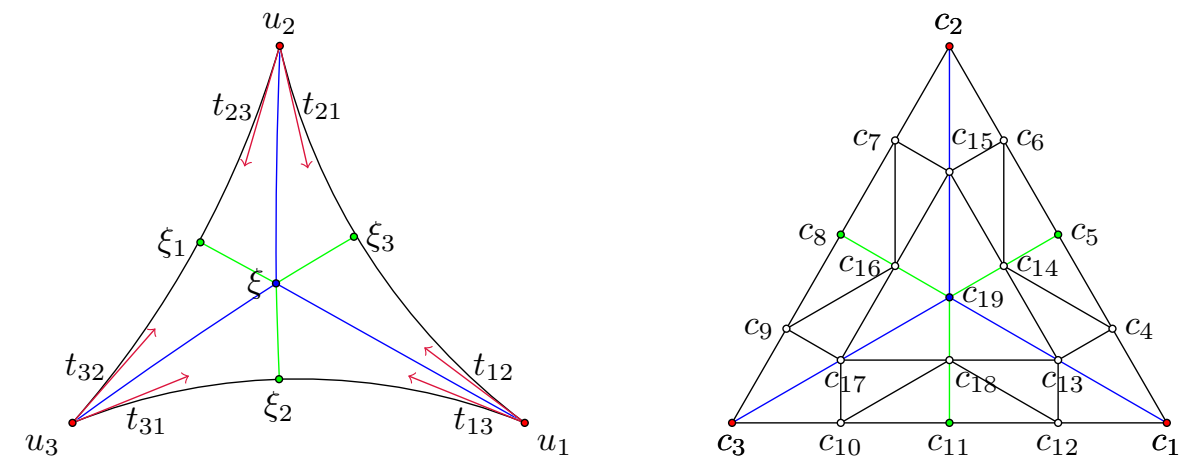

Figure 4: Powell-Sabin split of a triangle in $\Delta_{H}$ (left); Labeling of control points of a macro-element (right).

A reasonable way of estimating the directional derivatives is to require that the resulting interpolating spline has the so-called quadratic reproduction property. This means that if the vertices of the mesh $\Delta$ are incident on a quadratic homogeneous polynomial surface, then locally the spline must be identical to this surface. Such reproduction property is usually necessary for the interpolation scheme to have optimal approximation order. More precisely, suppose that we desire to estimate directional derivatives at a vertex $u_{1}$ of $\Delta_{H}$ and that $\mathcal{T}=\left\langle u_{1}, u_{2}, u_{3}\right\rangle \in \Delta_{H}$ is a triangle containing this vertex. To this end, we first find a best quadratic fit of the mesh points of $\Delta$ in the vicinity of $v_{1}$, the mesh point of $\Delta$ corresponding to vertex $u_{1}$. In particular, our objective is to find a quadratic parametric polynomial $q \in \pi_{2}$, parameterized by $b=\left(b_{1}, b_{2}, b_{3}\right)^{t}$, the barycentric coordinates w.r.t. the reference triangle $\mathcal{T}$, such that

$$
q\left(b\left(u_{1}\right)\right)=q\left((1,0,0)^{t}\right)=v_{1}
$$

and that

$$
\sum_{u \in \mathcal{U}_{1}}\|q(b(u))-v(u)\|_{2}^{2}=\sum_{u \in \mathcal{U}_{1}}\left\|\sum_{i+j+k=2} c_{i j k} B_{i j k}^{2}(u)-v(u)\right\|_{2}^{2}
$$

is minimized, where $\|\cdot\|_{2}$ denotes the Euclidean norm, and where $v(u)$ denotes the mesh point of $\Delta$ corresponding to vertex $u$. The sum above is taken over all vertices $\mathcal{U}_{1}$ of $\Delta_{H}$ that are in the vicinity of $u_{1}$. In our implementation, we considered the set $\mathcal{U}_{1}$ of all mesh points of $\Delta_{H}$ that either belong to a triangle of $\Delta_{H}$ containing $u_{1}$, or to a triangle sharing an edge with a triangle containing $u_{1}$. This guarantees 
that the size of $\mathcal{U}_{1}$ is at least 6 , the dimension of $\pi_{2}$, and that the above problem has a unique solution. Note that the minimization problem gives rise to three separate linear systems for the $x, y, z$-coordinates of the Bézier points $c_{i j k} \in \mathbb{R}^{3}$. Moreover, the resulting quadratic fit will not depend on which reference triangle $\mathcal{T}$ containing vertex $u_{1}$ we select or which particular representative $\left\langle u_{1}, u_{2}, u_{3}\right\rangle$ in the equivalence class of congruent triangles we consider for $\mathcal{T}$.

Once a local quadratic fit $q$ is found for vertex $u_{1}$, we can compute the sought-for directional derivatives in the direction of edges emanating from $u_{1}$ as follows. Let $\widehat{u_{1} u_{\ell}} \subset \mathcal{P}, \ell \neq 1$, be one such edge and suppose that $t_{1 \ell}$ is the unit tangent vector to the arc $\widehat{u_{1} u_{\ell}}$ at $u_{1}$, see Fig. 4 (left). The directional derivative $\delta_{1 \ell}$ of $q$ at $u_{1}$ in the direction $t_{1 \ell}$ is given as

$$
\delta_{1 \ell}:=D_{t_{1 \ell}} q\left(b\left(u_{1}\right)\right):=\left.\frac{d q(b(u(h)))}{d h}\right|_{h=0},
$$

where $u(h)$ is any smooth parametrization of the arc $\widehat{u_{1} u_{\ell}}$, such that $u(0)=u_{1}$ and $u^{\prime}(0)=t_{1 \ell}$. Using the chain rule, one obtains the following formula:

$$
\delta_{1 \ell}=b^{t}\left(t_{1 \ell}\right) \nabla_{b} q\left(b\left(u_{1}\right)\right)
$$

where $\nabla_{b}=\left(\partial_{b_{1}}, \partial_{b_{2}}, \partial_{b_{3}}\right)^{t}$ and $b\left(t_{1 \ell}\right)$ is the column vector of b.c.s of $t_{1 \ell}$ w.r.t. the reference triangle $\mathcal{T}=\left\langle u_{1}, u_{2}, u_{3}\right\rangle$. We note that since $q$ is quadratic, all three components of $\nabla_{b} q(b)$ are linear. As a result, one has $\nabla_{b} q\left(b\left(u_{1}\right)\right)=2\left(c_{200}, c_{110}, c_{101}\right)^{t}$, which are coefficients of the obtained quadratic fit $q$.

From now on, the construction of the Powell-Sabin macro-elements can proceed in a fully local way, as is standard in the classical setting [26]. First, Bézier coefficients associated with the edges of $\Delta_{H}$ are determined. For example, referring to Fig. 4 (right), for edge $\widehat{u_{1} u_{2}}$, these are coefficients $c_{1}, c_{2}, c_{4}, c_{5}, c_{6}$. The first two of these are readily determined by the interpolation conditions, $c_{1}=v_{1}, c_{2}=v_{2}$. The coefficients $c_{4}, c_{6}$ are obtained by the requirement that the directional derivatives in the direction of the edge are equal to $\delta_{12}, \delta_{21}$, respectively. For example, for the macro-element to interpolate the directional derivative $\delta_{12}$ at $u_{1}$, coefficient $c_{4}$ can be obtained as the solution of

$$
\delta_{12}=D_{t_{12}} p\left(b\left(u_{1}\right)\right)=2\left(b_{1} c_{1}+b_{2} c_{4}\right),
$$

where $b_{1}, b_{2}$ are the b.c.s of $t_{12}$ w.r.t. points $u_{1}$, $\xi_{3}$, i.e. $t_{12}=b_{1} u_{1}+b_{2} \xi_{3}$, and where $p$ is the quadratic polynomial associated with edge $\widehat{u_{1} \xi_{3}}$ (i.e. with coefficients $c_{1}, c_{4}, c_{5}$ ). Coefficient $c_{5}$ can now be determined by the $C^{1}$ conditions from $c_{4}, c_{6}$, namely

$$
c_{5}=b_{1}^{\prime} c_{4}+b_{2}^{\prime} c_{6}
$$

where now $b_{1}^{\prime}, b_{2}^{\prime}$ are the b.c.s of $\xi_{3}$ w.r.t. $u_{1}, u_{2}$, or $\xi_{3}=b_{1}^{\prime} u_{1}+b_{2}^{\prime} u_{2}$. All remaining Bézier coefficients, that are "interior" to a given triangle, say triangle $\left\langle u_{1}, u_{2}, u_{3}\right\rangle$ (i.e. coefficients $c_{13}, \ldots, c_{19}$ in Fig. 4 (right)), can be obtained from $C^{1}$-continuity conditions corresponding to adjacent subtriangles of the macro-element. For example, one has

$$
c_{13}=b_{1}^{\prime \prime} c_{1}+b_{2}^{\prime \prime} c_{4}+b_{3}^{\prime \prime} c_{12}, \quad c_{14}=b_{1}^{\prime} c_{13}+b_{2}^{\prime} c_{15}, \quad c_{19}=b_{1}^{\prime \prime \prime} c_{13}+b_{2}^{\prime \prime \prime} c_{15}+b_{3}^{\prime \prime \prime} c_{17},
$$

where $b_{1}^{\prime \prime}, b_{2}^{\prime \prime}, b_{3}^{\prime \prime}$ are the b.c.s of $\xi$ w.r.t. $u_{1}, \xi_{3}, \xi_{2}$ and $b_{1}^{\prime \prime \prime}, b_{2}^{\prime \prime \prime}, b_{3}^{\prime \prime \prime}$ are the b.c.s of $\xi$ w.r.t. $u_{1}, u_{2}, u_{3}$.

The above construction guarantees that adjacent macro-elements are $C^{1}$ continuous and thus so is the resulting composite spline surface. In particular, the $C^{1}$ continuity across the macro-edges (edges of $\Delta_{H}$ ) is due to the fact that the directional derivatives $\delta_{\ell \ell^{\prime}}$ are, by construction, compatible with a uniquely defined tangent plane at each vertex of $\Delta$.

\subsection{Interpolation by global energy minimization}

In the previous section we considered a local construction of a $C^{1}$ surface, composed of macro-elements. An alternative to the above approach is not to subdivide individual triangles of the mesh $\Delta$ and instead use the spline space $S_{n}^{m}(\Delta)$ directly, as defined in Section 2.3. Such an approach is quite robust in that it can 
be in principle applied for any parameters $m, n$ and can be used for a variety of data-fitting methods. In particular, if one wants to interpolate the vertices of the mesh $\Delta$ by a smooth spline, a standard possibility is to seek a solution of the following minimization problem: For given $m<n$, find a spline $s \in S_{n}^{m}(\Delta)$, such that $s\left(u_{i}\right)=v_{i}, i=1, \ldots, n_{V}$, and such that an energy- or "fairness" functional, $\mathcal{E}(s)$, is minimized. In the context of CAGD, this minimization is done to uniquely determine available degrees of freedom (i.e. any spline control points that are not readily set by the interpolation and smoothness conditions) and in such a way as to obtain a visually pleasing surface, free of artificial undulations not suggested by the geometry of the mesh $\Delta$.

The above interpolation problem can be formulated explicitly in terms of the Bézier coefficients of the sought-for spline. Let us suppose that these coefficients are collected in a vector $c=\left(c_{1}, \ldots, c_{M}\right)^{t}$, where $M=\operatorname{dim} S_{n}^{0}(\Delta)$ is as in (6). For convenience, we will assume that the coefficients are ordered such that the first $n_{V}$ entries, $c_{1}, \ldots, c_{n_{V}}$, of $c$ correspond to the Bézier points associated with vertices $v_{i}, i=1, \ldots, n_{V}$, of $\Delta$. Moreover, we assume that when two polynomial pieces join along an edge, the Bézier points along that edge are included in vector $c$ just once. With this convention, the interpolating spline $s$ can be determined as the minimizer of the energy functional $\mathcal{E}(s)$, subject to the constraints

$$
c_{i}=v_{i}, \quad i=1, \ldots, n_{V}
$$

and

$$
C c=0 \text {. }
$$

Here, $C$ is a $K \times M$ matrix that encodes the set of $C^{m}$-continuity conditions for $s$ across all edges of $\Delta$, specified in Proposition 4. These are linear homogeneous equations for the coefficients $c$.

Frequently, the energy functional can be expressed as a quadratic form:

$$
\mathcal{E}(c)=c^{t} Q c,
$$

where $Q$ is an $M \times M$ symmetric positive definite matrix. In that case, the interpolation problem reduces to finding a solution to the linear system:

$$
\left(\begin{array}{ccc}
Q & E^{t} & C^{t} \\
E & 0 & 0 \\
C & 0 & 0
\end{array}\right)\left(\begin{array}{l}
c \\
\mu \\
\nu
\end{array}\right)=\left(\begin{array}{l}
0 \\
v \\
0
\end{array}\right),
$$

where $E$ is the $n_{V} \times M$ matrix of the form $E=\left(I_{n_{V}} 0\right)\left(I_{n_{V}}\right.$ is the $n_{V} \times n_{V}$ identity matrix $), v=\left(v_{1}, \ldots, v_{n_{V}}\right)^{t}$, and $\mu=\left(\mu_{1}, \ldots, \mu_{n_{V}}\right)^{t}, \nu=\left(\nu_{1}, \ldots, \nu_{K}\right)^{t}$ are vectors of Lagrange multipliers associated with (11) and (12), respectively.

Some remarks on the above system are in order. If the full set of continuity conditions is considered, then the number of rows of $C$ is $K=(n+n-1+\cdots+n-m+1) n_{E}=\left(m n-\left(\begin{array}{c}m \\ 2\end{array}\right)\right) n_{E}$ since there are $n C^{1}$-continuity conditions per edge, $n-1$ equations for $C^{2}$ continuity, etc. It turns out that such matrix $C$ is rank deficient and therefore the system matrix in (14) is singular. This has been well known already in the context of ordinary splines [23] and even spherical splines [17]. However, the rank-deficiency of $C$ does not affect the consistency of the linear system. There are two basic approaches to attempt to solve such a system. Either one employs a system solver that can handle consistent singular linear systems or, alternatively, instead of $C$ one can consider a matrix with a reduced number of rows by eliminating dependent rows of $C$. The latter approach is tractable if $m$ is small compared to $n$ (in particular, if $n \geq 4 m+1$ or even $n \geq 3 m+2$ ), for in that case one can identify explicitly and in a local way redundant continuity conditions.

It is clear that the above-described approach is global, since all coefficients are the solution of a single linear system, which might be in fact quite large. However, the system is typically very sparse and hence system solvers taking advantage of the sparsity should be used. In particular, matrix $Q$ is sparse for a variety of energy functionals, including the one considered below, and so is the matrix $C$. For example, for $C^{1}$ continuity $(m=1)$ there are at most 4 nonzero entries in each row of $C$ and at most 7 such entries for $C^{2}$-continuous splines. 
Concerning the solvability of the system, because of the close resemblance of the splines considered here with the classical splines, we have reasons to believe that the system is uniquely solvable for any degree $n$ and smoothness $m$, as long as $n$ is sufficiently large relative to $m$. We plan to address this issue more rigorously elsewhere and, instead, we will content ourselves with pointing out that this claim is supported by our numerical experiments.

In the remainder of this subsection, we will discuss a suitable choice of energy for (13) that we have implemented and used in our experiments. Our starting point is the operator

$$
\mathcal{L}_{\mathcal{P}}=\partial_{x}^{2}+\partial_{y}^{2}+\sigma_{\mathcal{P}} \partial_{z}^{2}
$$

where

$$
\sigma_{\mathcal{P}}:= \begin{cases}1, & \text { if } \mathcal{P}=\mathcal{S} \\ 0, & \text { if } \mathcal{P}=\mathcal{A} \\ -1, & \text { if } \mathcal{P}=\mathcal{H}\end{cases}
$$

Thus, in the first two cases, $\mathcal{L}_{\mathcal{P}}$ is the standard Laplacian operator. We can now define the Laplace-Beltrami operator, $\mathcal{L}_{\mathcal{P}}^{*}$, by

$$
\mathcal{L}_{\mathcal{P}}^{*} f:=\left.\mathcal{L}_{\mathcal{P}} f\left(\cdot /\|\cdot\|_{\mathcal{P}}\right)\right|_{\mathcal{P}}
$$

which should hold for all sufficiently differentiable functions $f$ on $\mathcal{P}$. Thus, $\mathcal{L}_{\mathcal{P}}^{*} f$ is defined as the restriction to $\mathcal{P}$ of $\mathcal{L}_{\mathcal{P}}$ applied to the homogeneous extension of $f$ of degree 0 . Here $\|(x, y, z)\|_{\mathcal{P}}:=\sqrt{\sigma_{\mathcal{P}}\left(x^{2}+y^{2}\right)+z^{2}}$, so that $(x, y, z) /\|(x, y, z)\|_{\mathcal{P}} \in \mathcal{P}$, for all $(x, y, z) \in \mathbb{R}^{3}$ for which $\sigma_{\mathcal{P}}\left(x^{2}+y^{2}\right)+z^{2}>0$.

We now define the energy functional $\mathcal{E}(s)$ of a spline $s \in S_{n}^{m}(\Delta)$ as

$$
\mathcal{E}(s)=\sum_{\mathcal{T} \in \Delta_{H}} \int_{\mathcal{T}}\left(\mathcal{L}_{\mathcal{P}}^{*} s\right)^{2} d \tau
$$

where $d \tau$ is the Lebesgue measure on $\mathcal{P}$ corresponding to the standard metric on $\mathcal{P}$. We can now work out the entries of matrix $Q$ in (13). Clearly, if $s$ is a spline with coefficient vector $c$, the above functional has the form (13), with matrix entries

$$
Q_{\ell, \ell^{\prime}}=\sum_{\mathcal{T} \in \Delta_{H}} \int_{\mathcal{T}}\left(\mathcal{L}_{\mathcal{P}}^{*} B_{\ell}^{n, \mathcal{T}}\right)\left(\mathcal{L}_{\mathcal{P}}^{*} B_{\ell^{\prime}}^{n, \mathcal{T}}\right) d \tau, \quad \ell, \ell^{\prime}=1, \ldots, M .
$$

Here, the function $B_{\ell}^{n, \mathcal{T}}$ denotes the Bernstein basis polynomial corresponding to Bézier point $c_{\ell}$. If $c_{\ell}$ is not associated with $\mathcal{T}$, then $B_{\ell}^{n, \mathcal{T}}$ is understood to be zero. Thus, it is not difficult to see that $Q$ is sparse. Indeed, let $\ell, \ell^{\prime}$ be such that the Bézier points $c_{\ell}$ and $c_{\ell^{\prime}}$ correspond to interior Bézier points (i.e., points not associated with vertices or edges of $\Delta$ ) of two separate triangles $\mathcal{T}$ and $\mathcal{T}^{\prime}$. Then $Q_{\ell, \ell^{\prime}}=0$, since all summands in (17) are trivially zero.

To compute the non-zero entries of $Q$, we can use numerical quadrature. In our implementation, this has been done as follows. Consider a triangle $\mathcal{T}=\left\langle u_{1}, u_{2}, u_{3}\right\rangle \in \Delta_{H}$. Then the value of $\int_{\mathcal{T}}\left(\mathcal{L}_{\mathcal{P}}^{*} B_{\ell}^{n, \mathcal{T}}\right)\left(\mathcal{L}_{\mathcal{P}}^{*} B_{\ell^{\prime}}^{n, \mathcal{T}}\right) d \tau$ can be computed by parameterizing $\mathcal{T}$ by the planar triangle $\bar{T}$ with the same vertices as $\mathcal{T}$ [18]. In particular, a point $\bar{u} \in \bar{T}$ can be mapped to $u \in \mathcal{T}$ by the transformation

$$
u=\frac{A \bar{u}}{\|A \bar{u}\|_{\mathcal{P}}}
$$

where $A$ is the matrix whose columns are vectors $u_{1}, u_{2}, u_{3}$. One can show that the surface integral of an integrable function $f$ on $\mathcal{T}$ can be evaluated as

$$
\int_{\mathcal{T}} f(u) d \tau=\int_{\bar{T}} f\left(\frac{A \bar{u}}{\|A \bar{u}\|_{\mathcal{P}}}\right)\|N(\bar{u})\|_{\mathcal{P}} d \bar{u},
$$


where $N(\bar{u})$ is a normal vector of $\mathcal{P}$ at $u=A \bar{u} /\|A \bar{u}\|_{\mathcal{P}}$, such that $\|N(\bar{u})\|_{\mathcal{P}}=|\operatorname{det} A| /\|A \bar{u}\|_{\mathcal{P}}^{3}$. Thus, defining

we obtain

$$
h(\bar{u}):=\mathcal{L}_{\mathcal{P}}^{*} B_{\ell}^{n, \mathcal{T}}\left(\frac{A \bar{u}}{\|A \bar{u}\|_{\mathcal{P}}}\right) \mathcal{L}_{\mathcal{P}}^{*} B_{\ell^{\prime}}^{n, \mathcal{T}}\left(\frac{A \bar{u}}{\|A \bar{u}\|_{\mathcal{P}}}\right),
$$

$$
\int_{\mathcal{T}}\left(\mathcal{L}_{\mathcal{P}}^{*} B_{\ell}^{n, \mathcal{T}}\right)\left(\mathcal{L}_{\mathcal{P}}^{*} B_{\ell^{\prime}}^{n, \mathcal{T}}\right) d \tau=|\operatorname{det} A| \int_{\bar{T}} \frac{h(\bar{u})}{\|A \bar{u}\|_{\mathcal{P}}^{3}} d \bar{u} .
$$

The value of this integral can be computed by any standard numerical quadrature. We used a Gauss quadrature formula in our implementation.

The final issue that needs to be addressed is the evaluation of $\mathcal{L}_{\mathcal{p}}^{*} p$, where $p$ is a homogeneous polynomial of degree $n$, required in the computation of function $h$ above. Similarly to the spherical case [18], one can accomplish this by using the identity

$$
\mathcal{L}_{\mathcal{P}}^{*} p=\mathcal{L}_{\mathcal{P}} p-\sigma_{\mathcal{P}} n(n+1) p
$$

which holds for any homogeneous trivariate polynomial $p$ of degree $n$. This is a direct consequence of definitions (15) and (16). To evaluate the expression $\mathcal{L}_{\mathcal{p}} p$, we can view $p$ as a homogeneous trivariate function of the barycentric coordinates $b_{1}, b_{2}, b_{3}$ w.r.t. $\mathcal{T}$. By differentiating w.r.t. $b_{1}, b_{2}, b_{3}$ instead of $x, y, z$, we obtain

$$
\mathcal{L}_{\mathcal{P}} p=b^{t}\left(e_{1}\right) H_{b}(p) b\left(e_{1}\right)+b^{t}\left(e_{2}\right) H_{b}(p) b\left(e_{2}\right)+\sigma_{\mathcal{P}} b^{t}\left(e_{3}\right) H_{b}(p) b\left(e_{3}\right),
$$

where $e_{i}, i=1,2,3$ are the unit coordinate vectors and $H_{b}(p)$ is the Hessian matrix

$$
H_{b}(p):=\left(\frac{\partial^{2} p}{\partial b_{i} \partial b_{j}}\right)_{i, j=1}^{3} .
$$

\section{RAGS obtained from homogeneous splines}

Having discussed homogeneous splines in previous sections, it is natural to also consider rational versions of such functions. These turn out to be special cases of the rational geometric splines (or RAGS) introduced recently in [1], capable of representing smooth surfaces of arbitrary topological genus. To obtain RAGS from homogeneous splines, let $s: \Delta_{H} \rightarrow \mathbb{R}^{3}$ be a spline in $S_{n}^{m}(\Delta)$ and let $w: \Delta_{H} \rightarrow \mathbb{R}$ be a scalar homogeneous spline of degree $n$ and smoothness $C^{m}$ (i.e., an element of the scalar counterpart of $S_{n}^{m}(\Delta)$ ). We now consider a rational spline of the form $r_{H}=s / w$. In particular, for any triangle $\mathcal{T} \in \Delta_{H}$, denoting by $b(u)$ the b.c.s of $u \in \mathcal{T}$ w.r.t. $\mathcal{T}$, one can write for the restriction $r_{H}^{\mathcal{T}}$ of $r_{H}$ to $\mathcal{T}$ :

$$
r_{H}^{\mathcal{T}}(b(u))=\frac{\sum c_{i j k}^{T} B_{i j k}^{n, \mathcal{T}}(u)}{\sum w_{i j k}^{T} B_{i j k}^{n, \mathcal{T}}(u)}, \quad u \in \mathcal{T},
$$

where $c_{i j k}^{T} \in \mathbb{R}^{3}, w_{i j k}^{T} \in \mathbb{R}$ are the respective Bézier coefficients of $\left.s\right|_{\mathcal{T}}$ and $\left.w\right|_{\mathcal{T}}$, indexed by $T \in \Delta$, the triangle associated with $\mathcal{T} \in \Delta_{H}$, and where the summation is over all $i, j, k$ such that $i+j+k=n$ (see Fig. 5.) For the above rational function to be well-defined, we will assume that all coefficients $w_{i j k}^{T}$, which will be referred to as the weights, are positive. This is a sufficient condition for the spline $w$ to be positive. Clearly, since both splines $s, w$ are $C^{m}$ continuous, so is $r_{H}$. Moreover, $r_{H}^{\mathcal{T}}$ can be parameterized by the (flat) triangle $T$ and, consequently, it can be viewed as an ordinary rational function (i.e. the quotient of two algebraic bivariate polynomials). This can be seen by invoking the homogeneity of the Bernstein polynomials $B_{i j k}^{n, \mathcal{T}}$ as follows (cf. [1]):

$$
r_{H}^{\mathcal{T}}(b(u))=\frac{\sum c_{i j k}^{T}\left(b_{1}+b_{2}+b_{3}\right)^{n} B_{i j k}^{n, T}(v)}{\sum w_{i j k}^{T}\left(b_{1}+b_{2}+b_{3}\right)^{n} B_{i j k}^{n, T}(v)}=\frac{\sum c_{i j k}^{T} B_{i j k}^{n, T}(v)}{\sum w_{i j k}^{T} B_{i j k}^{n, T}(v)}=: r^{T}(\bar{b}(v)),
$$



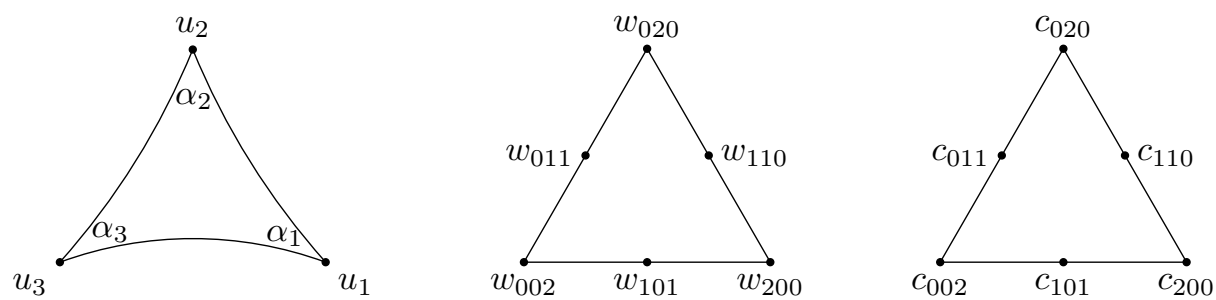

Figure 5: Labeling of Bézier coefficients in the case $n=2$.

where $\bar{b}(v):=\left(b_{1}, b_{2}, b_{3}\right)^{t} /\left(b_{1}+b_{2}+b_{3}\right)$ are the affine b.c.s of $v:=\bar{b}_{1} v_{1}+\bar{b}_{2} v_{2}+\bar{b}_{3} v_{3} \in T, v_{1}, v_{2}, v_{3}$ are the vertices of $T$, and where $B_{i j k}^{n, T}$ are ordinary Bernstein polynomials (i.e. functions of affine b.c.s $\bar{b}$.) Note that $\bar{b}(v)$ is well defined for all $v \in T$ since by our definition of b.c.s, $b_{1}+b_{2}+b_{3}=b_{1}(u)+b_{2}(u)+b_{3}(u)>0, u \in \mathcal{T}$. A consequence of the previous discussion is that $r_{H}$ can be viewed as a rational (algebraic) spline parameterized by the mesh $\Delta$, namely the rational spline $r: \Delta \rightarrow \mathbb{R}^{3}$ such that $\left.r\right|_{T}=r^{T}, T \in \Delta$. Moreover, this spline, viewed as the image of the mapping $r: \Delta \rightarrow \mathbb{R}^{3}$, is a $C^{m}$ surface. It follows that $r$ is a RAGS spline in the sense of [1]. However, it should be pointed out that while homogeneous geometry and the resulting homogeneous splines make it possible to construct RAGS, this is by no means the only way. We are currently exploring alternative methods of obtaining such splines.

Offhand, it is not clear whether a smooth positive spline $w$ needed for the above construction even exists. The answer is affirmative, at least in the case where $n$ is even, for then we can set $w$ to be identically equal to the constant function 1. This is because it is well known that the space of homogeneous polynomials of an even degree, restricted to $\mathcal{P}$, contains constants. As a consequence, in the even-degree case, constants are also elements of $S_{n}^{m}(\Delta)$, for all $m$. Note that this is not true for odd-degree polynomials, unless $\mathcal{P}=\mathcal{A}$. The existence of a smooth positive spline $w$ (with positive weights) for odd-degree splines is not guaranteed in general. However, we expect that such $w$ will typically exist if $n$ is "large enough" relative to $m$.

Next, we restrict ourselves to the even-degree case and derive a formula for the weights corresponding to the constant spline $w$. It will be sufficient to consider the case $n=2$ since for larger even values of $n$ the weights can be determined by degree elevation. More precisely, if $w_{i j k}$ are such that the quadratic polynomial $w=\sum_{i+j+k=2} w_{i j k} B_{i j k}^{2}$ is identically equal to 1 , we can obtain the weights corresponding to any other (even) degree $n$ by expanding out the right-hand side of identity $1=w^{n / 2}$.

Proposition 5. Let $w=\sum_{i+j+k=2} w_{i j k} B_{i j k}^{2}$ be a quadratic polynomial associated with a triangle $\mathcal{T} \subset \mathcal{P}$ with interior angles $\left\langle\alpha_{1}, \alpha_{2}, \alpha_{3}\right\rangle$, and suppose that $w$ is identically equal to one. Then

$$
w_{200}=w_{020}=w_{002}=1
$$

and

$$
w_{011}=\frac{\cos \alpha_{2} \cos \alpha_{3}+\cos \alpha_{1}}{\sin \alpha_{2} \sin \alpha_{3}}, \quad w_{101}=\frac{\cos \alpha_{1} \cos \alpha_{3}+\cos \alpha_{2}}{\sin \alpha_{1} \sin \alpha_{3}}, \quad w_{110}=\frac{\cos \alpha_{1} \cos \alpha_{2}+\cos \alpha_{3}}{\sin \alpha_{1} \sin \alpha_{2}} .
$$

Proof. The first set of identities follows from the interpolation property of the Bézier coefficients at the vertices of $\mathcal{T}$. As for the second set, we only prove the formula for $w_{110}$, i.e. the Bézier coefficient associated with the edge of $\mathcal{T}$ opposite to the third vertex of $\mathcal{T}$. Let us assume that a triangle with vertices $u_{1}, u_{2}, u_{3} \in \mathcal{P}$ is a representative of the equivalence class of triangles congruent to $\mathcal{T}$. To obtain $w_{110}$, the following must hold:

$$
w_{200} B_{200}^{2}(u)+w_{110} B_{110}^{2}(u)+w_{020} B_{020}^{2}(u)=b_{1}^{2}+2 w_{110} b_{1} b_{2}+b_{2}^{2}=1,
$$

for all $u \in \widehat{u_{1} u_{2}}$, where $b_{1}, b_{2}$ are the b.c.s of $u$ w.r.t. $u_{1}, u_{2}$. It will be sufficient to select any point $u$ in the interior of edge $\widehat{u_{1} u_{2}}$ (so that $b_{1} b_{2} \neq 0$ ) and solve for $w_{110}$. This will guarantee that equation (19) holds for three distinct values of $u$, and therefore it must be true for all $u$, given that constants are in the linear span 
of $B_{200}^{2}, B_{110}^{2}, B_{020}^{2}$. For simplicity, we set $u=\frac{u_{1}+u_{2}}{\left\|u_{1}+u_{2}\right\|_{\mathcal{P}}} \in \widehat{u_{1} u_{2}}$, which gives $b_{1}=b_{2}=1 /\left\|u_{1}+u_{2}\right\|_{\mathcal{P}}$, or, by (19),

$$
w_{110}=\frac{\left\|u_{1}+u_{2}\right\|_{\mathcal{P}}^{2}}{2}-1
$$

By an elementary calculation in the three separate cases, $\mathcal{P}=\mathcal{S}, \mathcal{A}, \mathcal{H}$, the previous expression simplifies to

$$
w_{110}= \begin{cases}\cos \left(d_{3}\right), & \mathcal{P}=\mathcal{S}, \\ 1, & \mathcal{P}=\mathcal{A}, \\ \cosh \left(d_{3}\right), & \mathcal{P}=\mathcal{H},\end{cases}
$$

where $d_{3}$ is the length of edge $\widehat{u_{1} u_{2}}$. The assertion of the proposition is now a consequence of formula $(7)$ and the analogous formula in the hyperbolic case.

In the context of rational splines it is customary to express individual patches of $r$, defined in (18), in the form

$$
r^{T}=\frac{\sum d_{i j k}^{T} w_{i j k}^{T} B_{i j k}^{n, T}}{\sum w_{i j k}^{T} B_{i j k}^{n, T}},
$$

where the $d_{i j k}^{T}:=c_{i j k}^{T} / w_{i j k}^{T} \in \mathbb{R}^{3}$ are called the control points of the rational spline. On account of the positivity of the weights, the control points are well defined. These control points are more convenient to work with since by (20), values of $r^{T}$ are obtained as convex combinations of such control points and hence they are geometrically more meaningful than the $c_{i j k}^{T}$ 's. In the usual way, the control points give rise to the so-called control net of $r$. By virtue of the convex hull property and the property that the control net is tangent to the corresponding surface at the vertices of the mesh, the control net mimics the behavior of the corresponding spline surface. In many other ways, working with RAGS is analogous to working with classical bivariate polynomial splines.

The set of rational splines $r$ considered here, corresponding to given fixed $m, n$, and $\Delta$, is not a linear space. However, one can obtain a linear space by fixing the weights $w_{i j k}^{T}, T \in \Delta$, i.e. by fixing the denominator of each rational patch $r^{T}$. In the setting considered here, in which RAGS are obtained from homogeneous splines, we propose to fix these weights, in the even-degree case, in accordance with Proposition 5 and the degree-elevation idea for degrees larger than two. Thus, one can think of the weights as being determined mainly by the combinatorial structure of the triangulation $\Delta$, i.e. by its topology, whereas the control points $d_{i j k}^{T}$ can be selected independently of the weights, with the aim to control the geometry of the resulting RAGS surface. Thinking of RAGS as linear spaces is of interest in connection with using such splines as finite elements for purposes of isogeometric analysis, i.e. for approximation as opposed to merely geometric modeling.

\section{Numerical experiments}

The following numerical examples illustrate RAGS surfaces obtained by methods described in previous sections. The surfaces displayed in Figs. 7 and 8 are examples of local and global interpolation by $C^{1}$ PowellSabin macro-elements for the mesh in Fig. 6 (left). In the former case, the required directional derivatives are estimated by the local approach presented in Section 4.1, which guarantees that the RAGS spline has the quadratic reproduction property. For the surface in Fig. 8, the derivatives are computed by requiring that the surface minimize the energy functional (13). As a consequence, the latter approach is global in nature and, as can be reasonably expected, it results in better aesthetic quality. Finally, the example in Fig. 9 is generated by the global interpolation method described in Section 4.2, employing degree-6 patches and $C^{2}$-continuity conditions. It turns out that each surface is tangent to its respective control net at the vertices of $\Delta$, in the same way as in the case of standard splines.

The illustrations in Figs. 7, 8, and 9 depict, from left to right, collections of individual surface patches, the final surface plots, the Gaussian curvature, and the zebra-line plots. 

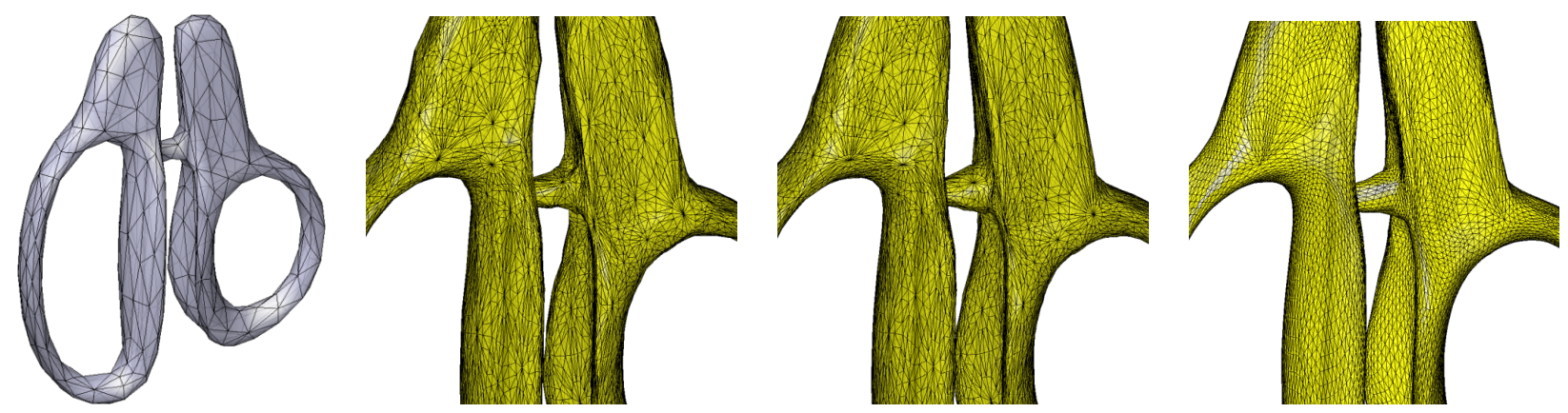

Figure 6: Initial triangulation (768 triangles) and details of control nets for the surfaces in Figs. 7, 8, and 9.
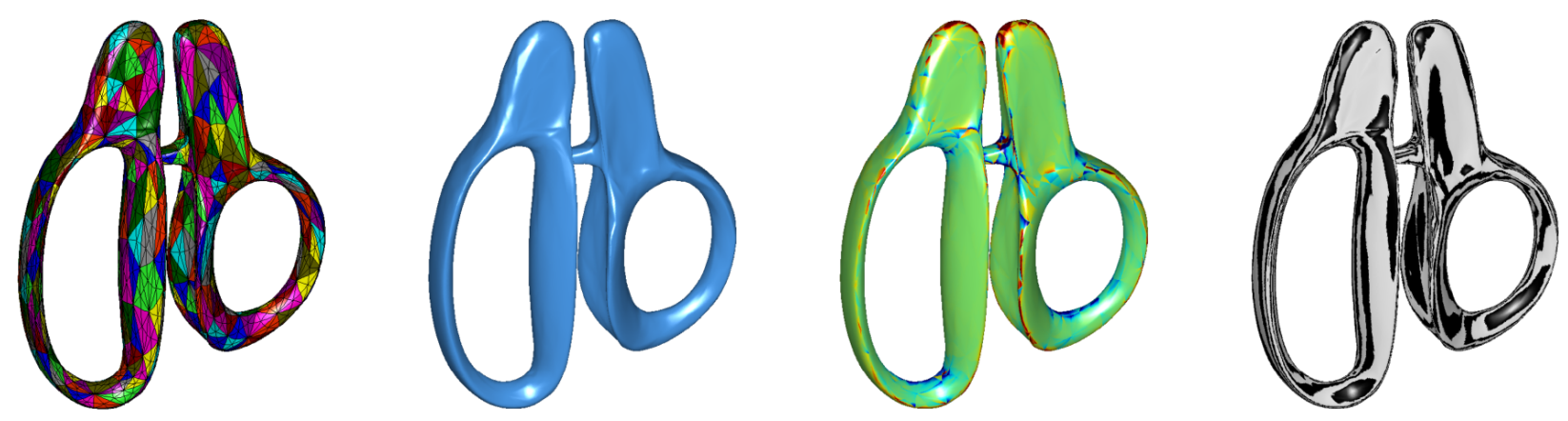

Figure 7: $C^{1}$ RAGS surface obtained by local interpolation with Powell-Sabin macro-elements.
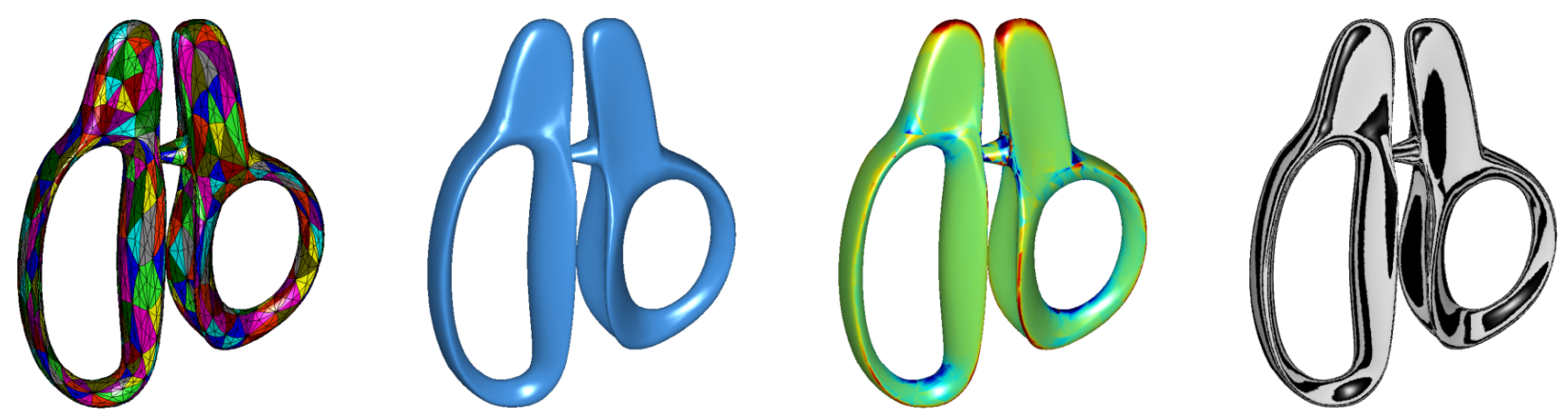

Figure 8: $C^{1}$ RAGS surface obtained by Powell-Sabin macro-elements and Laplacian energy minimization.

\section{Acknowledgements}

The first author acknowledges the support from the Italian INdAM National Group for Scientific Calculus (GNCS). She was also partially supported by the NSF grant DMS-1418742, by the Vanderbilt University International Research grant, and by the Marco Polo program of the University of Bologna. The second author was supported by the NSF grant DMS-1418742. We also wish to thank the anonymous referees for many detailed comments that helped us to improve the final manuscript.

\section{References}

[1] C. V. Beccari, D. E. Gonsor, M. Neamtu, RAGS: Rational geometric splines for surfaces of arbitrary topology, Comput. Aided Geom. Design 31 (2014) 97-110. 

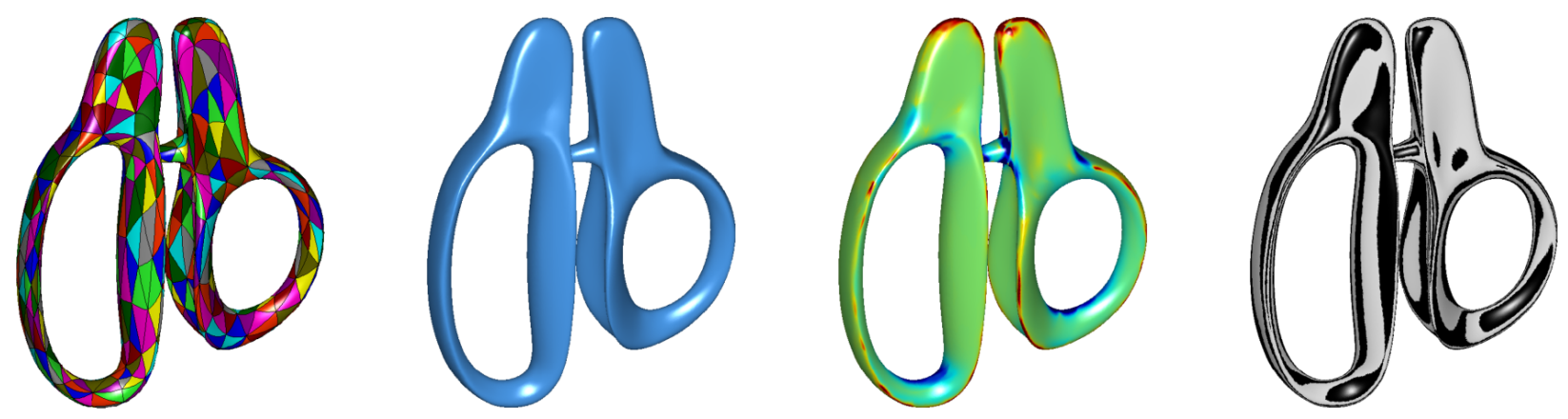

Figure 9: $C^{2}$ RAGS surface obtained by global interpolation with Laplacian energy minimization.

[2] C. Grimm, J. Hughes, Modeling surfaces of arbitrary topology using manifolds, in: Proceedings of SIGGRAPH 95, Computer Graphics Proceedings, Annual Conference Series, pp. 359-368.

[3] J. Navau, N. Garcia, Modeling surfaces from meshes of arbitrary topology, Comput. Aided Geom. Design 17 (2000) 643-671.

[4] G. Della Vecchia, B. Jüttler, M. Kim, A construction of rational manifold surfaces of arbitrary topology and smoothness from triangular meshes, Comput. Aided Geom. Design 25 (2008) 801-815.

[5] X. Gu, Y. He, M. Jin, F. Luo, H. Qin, S.-T. Yau, Manifold splines with a single extraordinary point, Computer-Aided Design 40 (2008) 676-690.

[6] E. Tosun, D. Zorin, Manifold-based surfaces with boundaries, Comput. Aided Geom. Design 28 (2011) 1-22.

[7] J. Warren, H. Weimer, Subdivision Methods for Geometric Design, Morgan Kaufmann, San Francisco, CA, USA, 2001.

[8] M. Sabin, Recent progress in subdivision: A survey, in: N. Dodgson, M. Floater, M. Sabin (Eds.), Advances in Multiresolution for Geometric Modelling, Springer, 2005, pp. 203-230.

[9] J. Peters, U. Reif, Subdivision Surfaces (Geometry and Computing), Springer, 2008.

[10] T. J. Cashman, Beyond Catmull-Clark? A survey of advances in subdivision surface methods, Comp. Graphics Forum 31 (2012) 42-61.

[11] T. W. Sederberg, J. Zheng, A. Bakenov, A. Nasri, T-splines and T-NURCCs, ACM Trans. Graph. 22 (2003) $477-484$.

[12] B. Li, H. Qin, Component-aware tensor-product trivariate splines of arbitrary topology, Computers \& Graphics 36 (2012) $329-340$

[13] M. Scott, R. Simpson, J. Evans, S. Lipton, S. Bordas, T. Hughes, T. Sederberg, Isogeometric boundary element analysis using unstructured T-splines, Comput. Methods Appl. Mech. Engrg. 254 (2013) 197-221.

[14] J. Peters, J. Fan, The projective linear transition map for constructing smooth surfaces, in: P. J. Philippe (Ed.), Shape Modeling International Conference SMI2010, IEEE Computer Society, 2010, pp. 124-130.

[15] J. Peters, M. Sarov, Polynomial spline surfaces with rational linear transitions, Computers \& Graphics 51 (2015) 43 - 51. International Conference Shape Modeling International.

[16] P. Alfeld, M. Neamtu, L. L. Schumaker, Bernstein-Bézier polynomials on spheres and sphere-like surfaces, Comput. Aided Geom. Design 13 (1996) 333-349.

[17] P. Alfeld, M. Neamtu, L. L. Schumaker, Dimension and local bases of homogeneous spline spaces, SIAM J. Math. Anal. 27 (1996) 1482-1501.

[18] P. Alfeld, M. Neamtu, L. L. Schumaker, Fitting scattered data on sphere-like surfaces using spherical splines, J. Comput. Appl. Math. 73 (1996) 5-43.

19] X. Liu, L. Schumaker, Hybrid Bézier patches on sphere-like surfaces, J. Comput. Appl. Math. 73 (1996) 157-172.

[20] H. Ferguson, A. Rockwood, Multiperiodic functions for surface design, Comput. Aided Geom. Design 10 (1993) 315-328.

[21] J. Wallner, Geometric Contributions to Surface Modeling, Ph.D. Thesis, Vienna University of Technology, 1996.

[22] H. Pottmann, J. Wallner, Spline orbifolds, in: A. Le Méhauté, C. Rabut, L. Schumaker (Eds.), Curves and Surfaces with Applications in CAGD, Vanderbilt University Press, Nashville, TN, 1997, pp. 445-464.

[23] M. Lai, L. Schumaker, Splines on Triangulations, Cambridge University Press, 2007.

[24] J. G. Ratcliffe, Foundations of Hyperbolic Manifolds, Graduate Texts in Math. 149, Springer-Verlag, New York, 2nd edition, 2007.

[25] M. S. Floater, Parametrization and smooth approximation of surface triangulations, Comput. Aided Geom. Design 14 (1997) 231-250.

[26] L. L. Schumaker, Spline Functions: Computational Methods, SIAM, 2015. 